\title{
De-protonation of Nickel Hydroxide by 200 kV Electron Irradiation
}

\author{
Graham J.C. Carpenter* and Zbigniew S. Wronski
}

Department of Natural Resources, Canada

*graham.carpenter@physics.org

\begin{abstract}
Previous studies of nickel hydroxide $\left(\mathrm{Ni}(\mathrm{OH})_{2}\right)$ powders have shown that either heating or mechanical grinding can result in complete de-hydroxylation, leading to conversion to nickel oxide (NiO). In both cases, this process appears to occur in one stage, without evidence for any intermediate compounds being formed. During studies of $\mathrm{Ni}(\mathrm{OH})_{2}$ powders for applications in the positive electrodes of Ni metal hydride (NiMH) rechargeable batteries, using transmission electron microscopy (TEM), we have observed significant changes caused by exposure to the highly energetic electron beam used for imaging and analysis. It is shown here, using electron energy-loss spectroscopy (EELS), that de-hydroxylation under electron irradiation occurs in two stages, with nickel oxy-hydroxide $(\mathrm{NiOOH})$ being formed at the intermediate stage.
\end{abstract}

Keywords: nickel hydroxide, de-hydroxylation, electron irradiation, EELS

\section{Introduction}

Since the second half of the twentieth century, Ni hydroxides have been used extensively in energy storage devices from rechargeable batteries to supercapacitors [1]. More recently, related compounds, in the form of layered metal hydroxides (LMH) and layered double (metal) hydroxides (LDH), have become important as novel inorganic functional materials.

Nickel hydroxide $\left(\mathrm{Ni}(\mathrm{OH})_{2}\right)$ shares its crystallographic structure with that of the well-known $\mathrm{Mg}(\mathrm{OH})_{2}$ brucite compound, with hexagonal packing of large $\mathrm{OH}^{-}$negative ions in alternate layers of octahedral sites filled by much smaller positive metal $\mathrm{Ni}$ ions, leading to the stacking of neutralcharged Ni-O layers. Bonding is anisotropic, with strong ionocovalent character within layers and more weak interaction between the layers. The relatively weak bonding between layers is the reason behind the easy shearing or rotation of layers, the tendency for which can be further promoted by partial substitution of $\mathrm{Ni}^{+2}$ by $\mathrm{Ni}^{+3}$ ions in the compound $\mathrm{NiOOH}$, or by transition metal atoms with distinct valences, such as $\mathrm{Co}^{+3}$. The latter has been applied to the development of high-energy, Co-doped batteries with $\mathrm{Ni}(\mathrm{OH})_{2}$ electrodes. Water molecules, or even other larger molecular species, can be efficiently intercalated between layers, in particular, in LDH. However, battery storage applications benefit more from the doping of layered $\mathrm{Ni}(\mathrm{OH})_{2}$ with $\mathrm{Li}^{+}$ions from a $\mathrm{KOH} / \mathrm{LiOH}$ electrolyte.

The hydrogen of the hydroxyl ions that belong to the interlayer space acquires a degree of mobility in the form of protons. In fact, $\mathrm{Ni}(\mathrm{OH})_{2}$ is a p-type semiconductor. Importantly, $\mathrm{LMH}$ can be exfoliated into single-lamellar nanosheets which, in tandem with the semiconducting properties, makes them promising candidates for a variety of applications in electronics, electro-catalysis, $\mathrm{CO}_{2}$ reduction, and hydrogen or oxygen evolution reactions on electrodes. Facilitating the de-protonation of an $\mathrm{OH}^{-}$group to $\mathrm{O}$ is of great importance for these applications. However, the processes occurring on the Ni layers during charge accumulation are not well understood.

In an earlier study [2], it was shown that electrochemical behavior involving the de-protonation of $\mathrm{Ni}(\mathrm{OH})_{2}$ powder could also be accomplished by either thermal or mechanical means, such as heating or mechanical grinding. Detailed work using a combination of scanning transmission electron microscopy (STEM) and differential scanning calorimetry (DSC) techniques showed that the transformation during heating occurred in one stage by means of the loss of $\mathrm{H}_{2} \mathrm{O}$ from the hydroxide layers. It was also shown that the same process could be induced in the hydroxide crystals by intensive mechanical milling [2,3] in a high-energy ball mill [4]. In addition, it was demonstrated that mechanical activation improves the mobility of hydrogen atoms (protons) in $\mathrm{Ni}(\mathrm{OH})_{2}$ crystals and hence improves the electrochemical performance of battery-grade hydroxide powders that are commonly used in NiMH rechargeable batteries [3].

During this study of $\mathrm{Ni}(\mathrm{OH})_{2}$ using TEM [2], it became apparent that the nature of this compound changed in the microscope when exposed to the intense, high-energy electron beam. An in situ experiment was, therefore, conducted using electron energy-loss near edge structures (ELNES) in an effort to understand the decomposition in more detail. Coincidentally, a separate study using EELS [5] was being conducted to detect changes that occurred in $\mathrm{Ni}(\mathrm{OH})_{2}$ during charge/discharge cycling of a positive $\mathrm{NiMH}$ electrode in which the active mass was nickel hydroxide $\mathrm{Ni}(\mathrm{OH})_{2}$. The results of that investigation provided new information on the near-edge structure of the $\mathrm{O}-\mathrm{K}$ core-loss edge, which proved to cast light on the process of de-protonation of $\mathrm{Ni}(\mathrm{OH})_{2}$ layers under electron irradiation.

\section{Techniques}

The $\mathrm{Ni}(\mathrm{OH})_{2}$ powder was in the form of porous spheres, with diameters up to $10 \mu \mathrm{m}$, that were made up of an agglomerate of thin platelets on the nanometer scale [2]. Specimens for TEM were prepared by embedding a sample of the powder in resin and cutting sections, $\sim 40 \mathrm{~nm}$ in thickness, using an ultramicrotome [6]. The specimens were examined in an FEI CM20FEG (field emission) TEM/STEM equipped with a Gatan Imaging Filter model 678, which permitted EELS spectroscopy at a resolution (FWHM) as low as $1 \mathrm{eV}$.

\section{Results}

The progress of the transformation under the electron beam is shown in the ELNES spectra of Figure 1, using conditions of slow de-hydroxylation under a defocused electron beam, which also had the effect of averaging the crystallographic orientations of the nanocrystals. The initial and final spectra were readily identified as $\mathrm{Ni}(\mathrm{OH})_{2}$ and $\mathrm{NiO}$, 


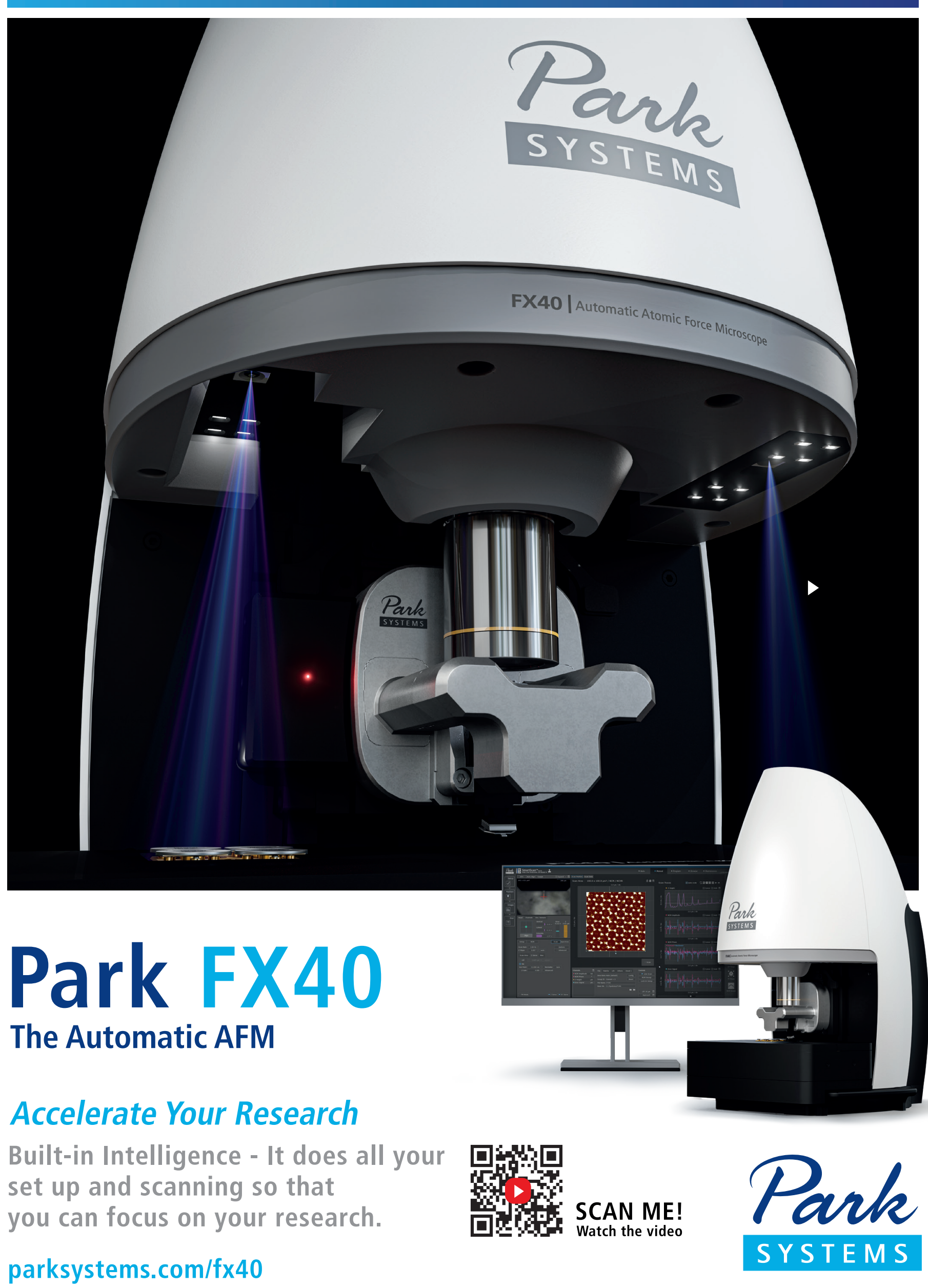




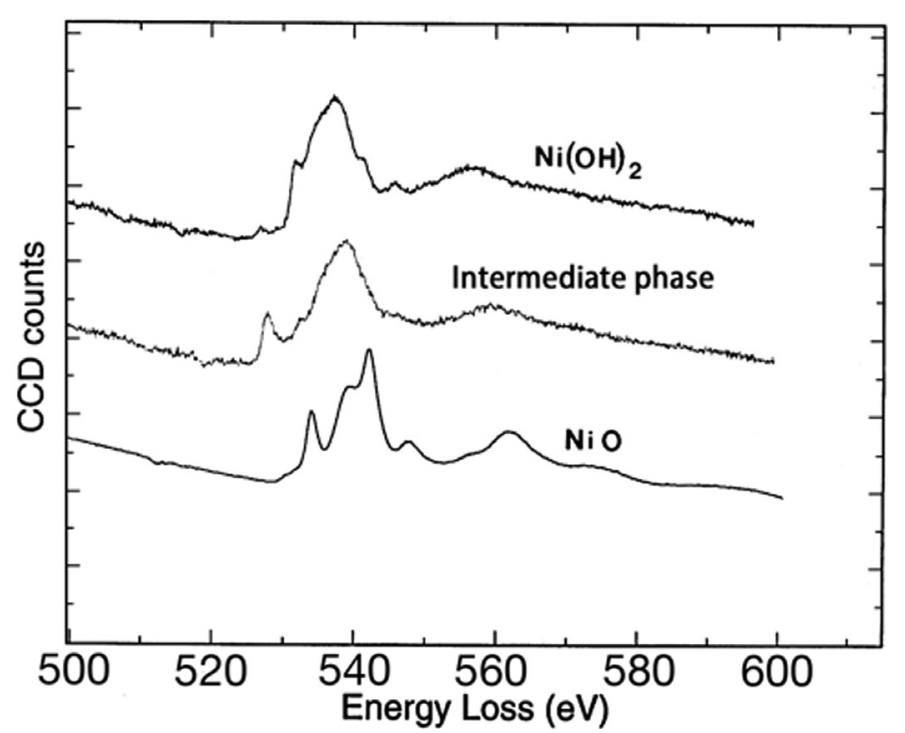

Figure 1: EELS spectra showing an intermediate stage in the transition from $\mathrm{Ni}(\mathrm{OH})_{2}$ to $\mathrm{NiO}$ during high-energy electron irradiation in the electron microscope.

respectively, by comparing them with the near-edge structure of those collected previously as reference spectra from pure reactant-grade powders [7]. It should be noted that compared to the reference spectra, additional fine structure is visible in the spectra of Figure 1 because they were collected under conditions of enhanced energy resolution.

It is clear from Figure 1 that a distinct intermediate stage exists, for which no comparable reference spectra could be found in the published literature. However, during a TEM investigation of the changes in a positive nickel electrode caused by charge/discharge cycling, where the active mass was $\mathrm{Ni}(\mathrm{OH})_{2}$, an ELNES spectrum was obtained for an electrode in the charged condition [5] (Figure 2). As discussed in more detail in the Discussion section, the effect of charging this form of electrode causes the $\mathrm{Ni}(\mathrm{OH})_{2}$ to be electrochemically oxidized to the well-established nickel oxy-hydroxide phase, $\mathrm{NiOOH}$. A comparison of the peak positions in the fine structure of the EELS spectrum, particularly that at $527 \mathrm{eV}$, shows clearly that the spectrum from the intermediate stage of de-protonation must indeed correspond to that from $\mathrm{NiOOH}$. Further evidence for this designation was obtained from a high-resolution X-ray photoemission spectroscopy (XPS) study, which revealed an extra peak at a comparable energy $(\sim 529 \mathrm{eV})$ in the oxygen $\mathrm{Ols}$ edge for a $\mathrm{NiOOH}$ layer forming on Ni metal exposed to oxidation by water vapor [8]. A similar peak was observed in a de-convoluted profile for solution processed $\mathrm{NiOx}$ for photovoltaic applications [9].

To eliminate any doubt that the intermediate spectrum could have been caused by the overlap of the hydroxide and oxide phases, the two spectra were summed for comparison purposes.

This was not possible in a direct manner because the original digital data could no longer be accessed. The printed output for the initial hydroxide phase and the final oxide was therefore re-digitized using the application WebPlotDigitizer ${ }^{\circ}$ (Automeris) and the data summed and re-plotted using Excel. The results, displayed in Figure 3, show quite clearly that

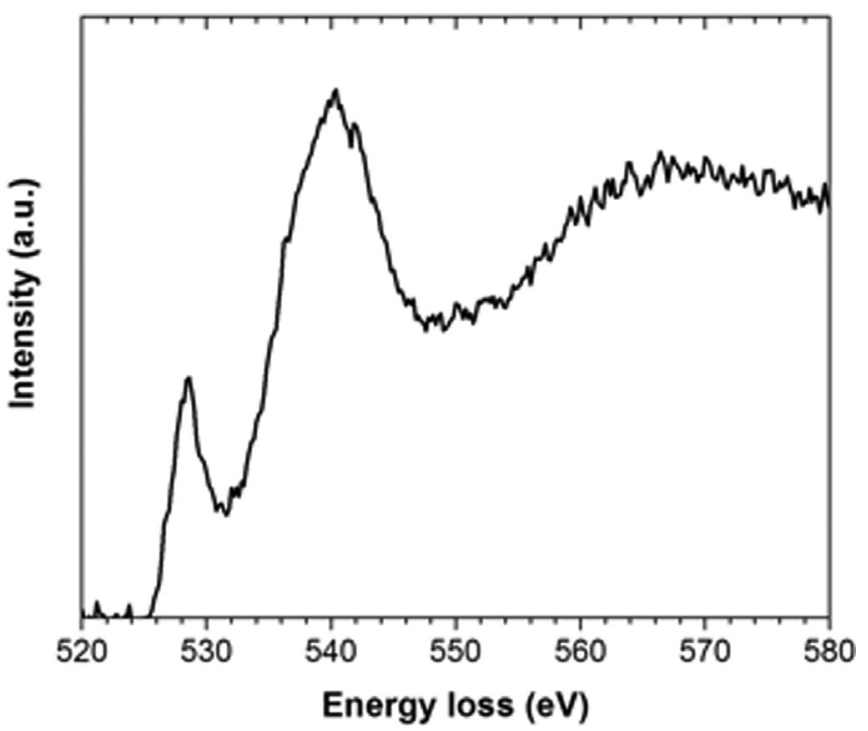

Figure 2: EELS spectrum from the active mass of a $\mathrm{NiMH}$ electrode in the charged condition after charge/discharge cycling. Reproduced with kind permission from Microscopy Today.

the spectrum from the intermediate phase is quite different from the sum of the spectra from the start and end of the de-protonation experiment.

\section{Discussion}

This is the first time the process of de-protonation of dry $\mathrm{Ni}(\mathrm{OH})_{2}$ has been observed to occur via an intermediate stage, with the formation of $\mathrm{NiOOH}$, apart from by electro-chemical

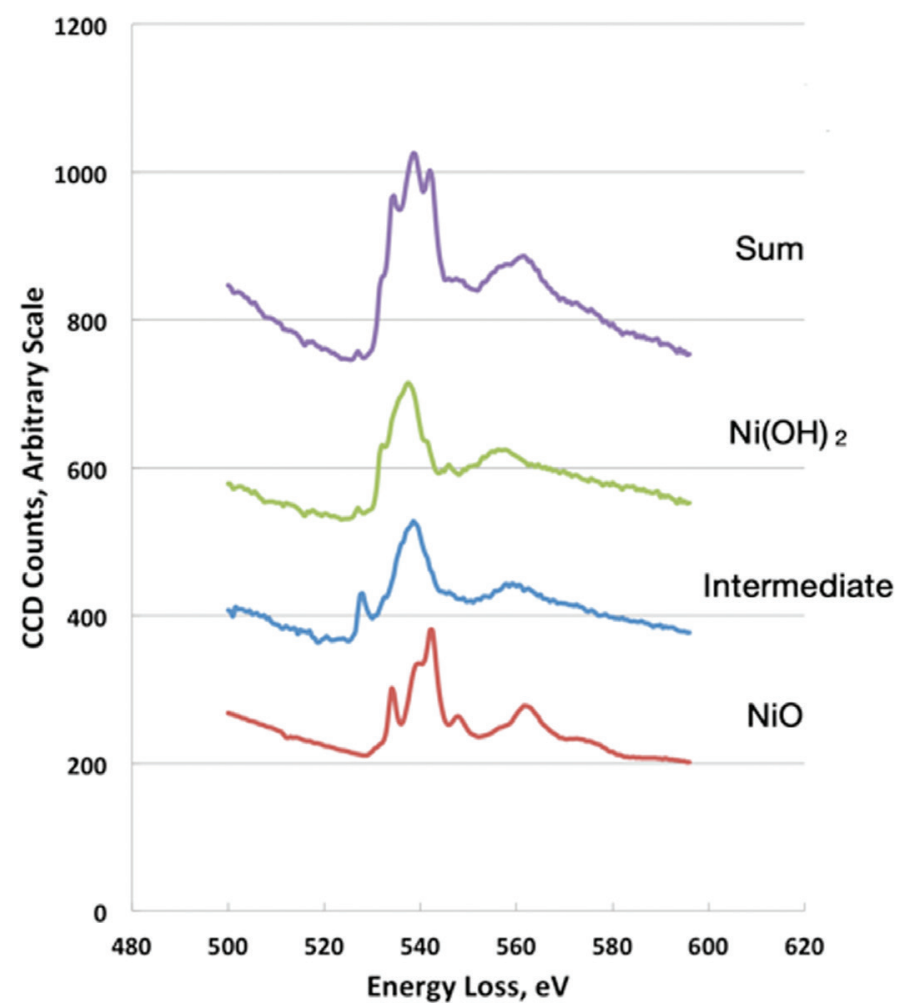

Figure 3: The data of Figure 1, re-plotted to allow comparison of the intermediate spectrum with the sum of the spectra for $\mathrm{Ni}(\mathrm{OH})_{2}$ and $\mathrm{NiO}$. 
means. Based on thermal heating experiments conducted in a DSC [2], it was established that full de-protonation of $\mathrm{Ni}(\mathrm{OH})_{2}$ proceeds by de-hydroxylation in a single stage by the loss of water molecules during heating at $\sim 300^{\circ} \mathrm{C}$ (equation 1):

$$
\mathrm{Ni}(\mathrm{OH})_{2}=\mathrm{NiO}+\mathrm{H}_{2} \mathrm{O}
$$

It was also shown that $\mathrm{NiO}$ could be produced by mechanical grinding of dry $\mathrm{Ni}(\mathrm{OH})_{2}$ powders, also with no evidence for an intermediate stage [2].

In contrast, electrochemical charge-discharge cycling of $\mathrm{Ni}(\mathrm{OH})_{2}$ in a fresh battery electrode does create $\mathrm{NiOOH}$ on each cycle of charge [3], according to equation 2.

$$
\beta-\mathrm{NiOOH}+\mathrm{H}_{2} \mathrm{O}+\mathrm{e}^{-} \leftrightarrows \beta-\mathrm{Ni}(\mathrm{OH})_{2}+\mathrm{OH}
$$

(forward is discharge, reverse is charge)

This reaction is the essential process by which the positive $\mathrm{Ni}$ electrode operates in a rechargeable NiMH battery. The charge cycle of de-hydroxylation operates from the recombination of a hydroxyl ion from a dissociated water molecule and hydrogen coming from the de-protonated hydroxide.

In this research we found that $\mathrm{NiOOH}$ is produced in the dry $\mathrm{Ni}(\mathrm{OH})_{2}$ powder during electron irradiation in the TEM, with an extra peak in the ELNES spectrum at about $527 \mathrm{eV}$. We can attribute this peak to $\mathrm{NiOOH}$ because its near-edge energy $527 \mathrm{eV}$ is close to $529 \mathrm{eV}$ for the binding energy of $\mathrm{O}^{2-}$ in the O1s peak [8]. Moreover, the careful perusal of the spectrum suggests that the $\mathrm{NiOOH}$ coming from the irradiation process in this study may be somewhat disordered. This is shown by the substantial intensity of the O1s peak of the oxide group $\mathrm{O}^{2-}$, as compared to the very weak peak characteristic of the hydroxyl $\mathrm{OH}^{-}$group, which should be visible at approximately $231 \mathrm{eV}$ for the disordered $\gamma$ form of $\mathrm{NiOOH}$ [5].

For the $\mathrm{NiOOH}$ intermediate phase to occur in dry $\mathrm{Ni}(\mathrm{OH})_{2}$, the decomposition process would have to occur by the following reaction (equation 3):

$$
2 \mathrm{Ni}(\mathrm{OH})_{2}=2 \mathrm{NiOOH}+2 \mathrm{H}
$$

This reaction would require the diffusion of protons to the surfaces of the hydroxide crystals followed by the recombination of the hydrogen atoms and the release of hydrogen (equation 4):

$$
\mathrm{H}+\mathrm{H}=\mathrm{H}_{2}
$$

For the intermediate phase to occur (as in a fresh electrode) in dry $\mathrm{Ni}(\mathrm{OH})_{2}$ under irradiation by $200 \mathrm{kV}$ electrons in a TEM, it is likely that this is a result of the experiment being carried out in situ using a very thin specimen. Thus, we would expect that the hydrogen atoms could readily diffuse out through the nearby specimen surfaces and recombine into $\mathrm{H}_{2}$, to be lost in the vacuum of the microscope. If this experiment were carried out using thicker specimens, for example, in a high-voltage electron microscope, it is possible that recombination of the $\mathrm{H}$ ions would result in the formation of small hydrogen bubbles within the thin foil specimen.

The de-protonation process under the electron beam leads to an intermediate phase, one which is not created from $\mathrm{Ni}(\mathrm{OH})_{2}$ by either heating or mechanical grinding. We conclude that the dissociation reaction to form protons is caused primarily by ionization damage from the $200 \mathrm{kV}$ charged electrons, which can readily supply the bond-breaking energy needed for the transformation.

\section{Acknowledgements}

It is a pleasure to thank Prof. David McComb of the Ohio State University for invaluable discussions and advice on optimizing the EELS spectra, when he was a summer visitor at Natural Resources Canada (Ottawa).

\section{References}

[1] J McBreen, "Nickel Hydroxides" in Handbook of Battery Materials (1997) JO Besenhard, ed., Wiley-VCH, Germany. https://www.osti.gov/servlets/purl/757095.

[2] GJC Carpenter and ZS Wronski, Nanostruct Mat 11 (1999) https://doi.org/10.1016/S0965-9773(99)00020-3.

[3] ZS Wronski et al., J Nanosci Nanotech 9 (2009) https://doi .org/10.1166/jnn.2009.M09.

[4] C Suryanarayana, Prog Mater Sci 46 (2001) https://doi .org/10.1016/S0079-6425(99)00010-9.

[5] GJC Carpenter et al., Microscopy Today 29 (2021) https:// doi.org/10.1017/S1551929521000869.

[6] MT Shehata and GJC Carpenter, Proc $28^{\text {th }}$ Ann Tech Mtg IMS Symp (1996) DW Stevens et al., eds., ASM International, Materials Park, OH. ISBN-13: 978-0871705709.

[7] GJC Carpenter and Z Wronski, Microsc Microanal 21 (2015) https://doi.org/10.1017/S1431927615015470.

[8] BP Payne et al., J Electron Spectrosc Rel Phenomena 175 (2009) https://doi.org/10.1016/j.elspec.2009.07.006.

[9] EL Ratcliff et al., Chem Mater 23 (2011) https://doi .org $/ 10.1021 / \mathrm{cm} 202296 \mathrm{p}$.

\section{MRS-6}

\section{We are ISO-9000 certified and ISO-17025 accredited Microscopy Calibration Standard}

Now you can calibrate better from $1,000 \mathrm{X}$ to $1,000,000 \mathrm{X}$ !

This is our fifth generation, traceable, magnification reference standard for all types (SEM, FESEM, Optical, STM, AFM, etc.) of microscopy. The MRS-6 has multiple $X$ and $Y$ pitch patterns ranging from $80 \mathrm{~nm}( \pm 3 \mathrm{~nm})$ to $2 \mu \mathrm{m}$ and 3 bar targets from $80 \mathrm{~nm}$ to $3 \mu \mathrm{m}$. There is also a STM test pattern. Definition of the $80 \mathrm{~nm}$ pitch pattern is excellent.

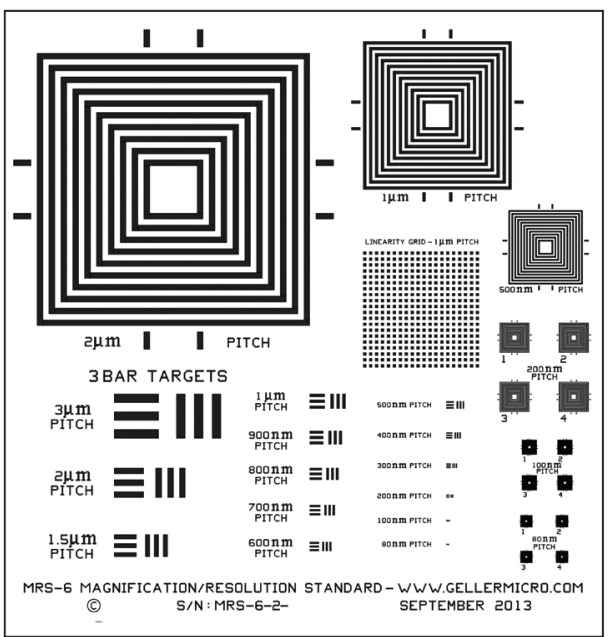

\section{GELLER} MICROÅNALYTICAL LABORATORY, Inc.

426e BOSTON ST., TOPSFIELD, MA 01983-1216 TEL: 978 887-7000 FAX: 978-887-6671 www.GellerMicro.com 